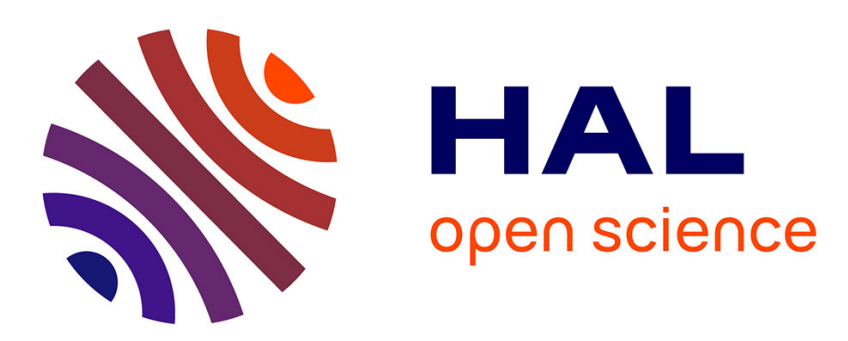

\title{
Structure Factor of EuCl_3 Aqueous Solutions via Coupled Molecular Dynamics Simulations and Integral Equations
}

Raj-Kumar Ramamoorthy, Maximilien Levesque, Luc Belloni, David Carriere

\section{- To cite this version:}

Raj-Kumar Ramamoorthy, Maximilien Levesque, Luc Belloni, David Carriere. Structure Factor of EuCl_3 Aqueous Solutions via Coupled Molecular Dynamics Simulations and Integral Equations. Journal of Physical Chemistry B, 2020, 124, pp.1787-1793. 10.1021/acs.jpcb.9b11537 . cea-02476063

\section{HAL Id: cea-02476063 https: / hal-cea.archives-ouvertes.fr/cea-02476063}

Submitted on 12 Feb 2020

HAL is a multi-disciplinary open access archive for the deposit and dissemination of scientific research documents, whether they are published or not. The documents may come from teaching and research institutions in France or abroad, or from public or private research centers.
L'archive ouverte pluridisciplinaire HAL, est destinée au dépôt et à la diffusion de documents scientifiques de niveau recherche, publiés ou non, émanant des établissements d'enseignement et de recherche français ou étrangers, des laboratoires publics ou privés. 


\title{
Structure Factor of $\mathrm{EuCl}_{3}$ Aqueous Solutions via Coupled
}

\section{Molecular Dynamics Simulations and Integral Equations}

\author{
Raj Kumar Ramamoorthy ${ }^{1, \dagger, *}$, Maximilien Levesque ${ }^{2}$, Luc Belloni ${ }^{1}$, and David \\ Carrière $^{1, *}$ \\ ${ }^{1}$ Université Paris-Saclay, CEA, CNRS, NIMBE, LIONS, 91191, Gif-sur-Yvette, \\ France
}

${ }^{2}$ PASTEUR, Département de chimie, École normale supérieure, PSL University, Sorbonne Université, CNRS, 75005 Paris, France

February 4, 2020

\section{Abstract}

Identifying the structure of an aqueous solution is essential to rationalize various phenomena such as crystallization in solution, chemical reactivity, extraction of rare earth elements, etc.. Despite this, the efforts to describe the structure of an aqueous solution have been hindered by the difficulty to retrieve structural data both from experiments and simulations. To overcome this, first, undersaturated $\mathrm{EuCl}_{3}$ aqueous solutions of concentrations varying from 0.15 to $1.8 \mathrm{~mol} / \mathrm{kg}$ were studied using X-ray scattering. Second, for the first time, the theoretical X-ray signal of $1.8 \mathrm{~mol} / \mathrm{kg} \mathrm{EuCl} 3$ aqueous solution was simulated, with precise details for the complete range of scattering vectors using coupled molecular dynamics and hypernetted chain integral equations, and satisfactorily compared with the $1.8 \mathrm{~mol} / \mathrm{kg}$ experimental X-ray scattering signal. The theoretical calculations demonstrate that the experimental structure factor is dominated by the $\mathrm{Eu}^{3+}-\mathrm{Eu}^{3+}$ correlations.

\footnotetext{
${ }^{\dagger}$ Current address: Laboratoire de Génie Chimique, Université de Toulouse, CNRS, INPT, UPS, Toulouse, France.

${ }^{*}$ Corresponding authors: rajrk37@gmail.com and david.carriere@cea.fr; Phone: +33 (0)1 69085489 (D. C.)
} 


\section{Introduction}

At large scales, aqueous solutions of ions are homogeneous and isotropic but at the molecular scale, they show complex solvent/solute distributions stemming from the mutual interactions between the ions and the water molecules, not to mention possible speciation effects. Describing this complex distribution of ions in water, and the structural correlations (ion-ion and ion-water), is essential to rationalize a broad range of phenomena such as crystallization in solution ${ }^{1}$, extraction of rare earth elements ${ }^{2}$, chemical reactivity ${ }^{3}$, separation process ${ }^{4}$, appearance of first sharp diffraction peak (or pre-peak at very short angle) $\frac{5}{5}$, etc.. Indeed, elucidating the structure at the scale of the ions and molecules is the premise to anticipate the kinetics of diffusion and reaction via the effective size (ions plus hydration shells) and accessibility of the species; and furthermore, at large concentrations, it allows rationalization of the first steps towards crystallization, involved in ion recovery processes. The structural correlations in a given solution can be determined by using $\mathrm{X}$-ray scattering,, 6$]$ that provide atomic-range structural information, directly in solution, without perturbing the solution. However, the information obtained through this technique is the total scattered intensity, which provides a global information that integrates thus loses all details about the ion-ion and ion-water correlations.

The identification of the individual contributions of the solutes and solvent in the scattering pattern would benefit from comparison with theoretical patterns. As usual in statistical mechanics of liquids and solutions, two competing routes are possible. (a) On the one hand, numerical simulations such as Monte-Carlo $(\mathrm{MC})^{\sqrt[8]{8}}$ or molecular dynamics $(\mathrm{MD})^{\sqrt[9]{9}}$ provide in principle an exact treatment of the multibody correlations and should be the method of choice for calculating the exact pair distribution functions (PDFs) for given pair potentials $v_{i j}(r, \Omega)$ that depend on the separation $(r)$ and the relative orientation $(\Omega)$. The existence of strong coulombic couplings, anisotropic correlations, hydrogen bonding, hydration layers, etc. makes the computer simulation quite difficult, especially in presence of multivalent ions and at low concentrations. In practice, beyond the need for long and costly simulations, beyond the presence of statistical uncertainties and systematic corrections, the answer is only partial due to the finite size $L$ of the simulation cell: the nonnegligible tail of the PDFs is lacking and, equivalently, the domain $q<2 \pi / L$ is unreachable in the Fourier space, rendering the comparison with experimental data incomplete and quite frustrating. On the other hand, (b) the formalism based on the Ornstein-Zernike (OZ) equation requires the use of approximated closures or integral equations. Unfortunately, the standard hypernetted chain (HNC) equation closure which gives reasonable results, at least qualitatively, for pure water and monovalent salts, fails completely and has no numerical solution for the multi-valence ionic mixtures. Therefore, to achieve best possible simulation signal for a multi-valence ionic solution, we will need to combine both routes a) and b) by completing the MD data available at short distances 
$r<r_{\max } \approx L / 2$ (formerly cleaned of finite-size corrections ${ }^{10}$ ) with the HNC approximation valid at least at large distances, $r>r_{\max }$. This procedure, originally proposed by Verlet for simple Lennard-Jones (LJ) fluids ${ }^{11}$, has been recently extended to pure liquid water ${ }^{12}$ and shown to be a very powerful approach for deriving exact anisotropic correlations at all distances $(r)$, orientations $(\Omega)$ and scattering vectors $(q)$.

Besides calculating the structure factors for each pair potential, the close matching of computed scattering signal with the experimental signal will be depending on (i) how close the predicted model coincides with the real situations and (ii) the wise mathematical manipulation of the computed partial structure factors. Further, it is known from literature that the presence of solute (nature and oxidation state(s)) in water would modify the existing structure ${ }^{\sqrt{9113}}$ and in turn, its structural correlation correlation. Therefore, a mere addition of pure water structure factor, along with the other components, would not be sufficient to reproduce the recorded experimental X-ray scattering signal. Thus, estimating the total scattering signal would be challenging.

Keeping all these questions/challenges in our minds, $\mathrm{EuCl}_{3}$ aqueous solution was selected for theoretical (to check the consistency of coupled MD/HNC equations on the tri-valence aqueous solution) and experimental reasons (to reveal the possible structural correlation of rare earth ions, during the crystallization ${ }^{14}$ and to understand more complex solutions involved in industrial recovery processes $\frac{15}{15}$ ). In this study, we use X-ray scattering to examine a under-saturated, speciation-free, multivalent, $\mathrm{EuCl}_{3}$ aqueous solutions of concentrations varying from 0.15 to $1.8 \mathrm{~mol} \mathrm{~kg}^{-1}$. Simultaneously, for the first time, coupled MD simulation and HNC integral equations were performed, for the highest concentration of $\mathrm{EuCl}_{3}$ solution $(1.8 \mathrm{~mol} / \mathrm{kg})$, to compute the various structural correlations in the aqueous solution for the recorded $q$-range. Finally, these structural correlations were summed to obtain the best theoretical scattering signal and satisfactorily compared with the experimental scattering signal of $1.8 \mathrm{~mol} / \mathrm{kg} \mathrm{EuCl} \mathrm{E}_{3}$ aqueous solution.

\section{Materials and Methods}

\subsection{Experimental section}

As received $\mathrm{EuCl}_{3}$ (from Sigma-Aldrich, 99.99\%) was used without further purification. The appropriate amount of $\mathrm{EuCl}_{3}$ was dissolved in MilliQ water to obtain the required concentrations of $\mathrm{EuCl}_{3}$ aqueous solutions i.e., $0.15,0.33,0.72$ and $1.8 \mathrm{~mol} / \mathrm{kg}$. Small and wide X-ray scattering (SAXS/WAXS) were performed on a home-made apparatus in the scattering vector range $2.3 \times 10^{-2} \AA^{-1} \leq q \leq 3 \AA^{-1} 16$. The X-ray source (rotating molybdenum anode, $\lambda=0.709 \AA$ ) is collimated via an Osmic mirror through two hybrid slits $(1 \mathrm{x} 1 \mathrm{~mm})$. The beam scattered through the sample (kapton tube, $1 \mathrm{~mm}$ diameter) was collected on a Mar 345 image plate, with a sam- 
ple-detector distance of $72 \mathrm{~cm}$ (calibrated with tetradecanol). The detector count is normalized to differential cross-section per volume with $3 \mathrm{~mm}$ Lupolen as a secondary reference $\left(I_{\max }=6 \mathrm{~cm}^{-1}\right)$,

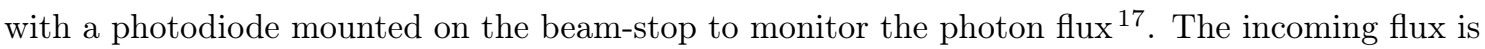
found to be $9 \times 10^{7}$ photons per second. To obtain an absolute intensity of sample, X-ray scattering signal was subtracted for various background corrections such as dark current, sample holder and $q$-dependent geometrical factor. The $q$-dependent geometrical correction factor was obtained by comparing the X-ray scattering signal of water recorded at lab and ID02 beamline (ESRF) 18 .

\subsection{Computation methods}

\subsubsection{Pair potentials}

The pair potentials or force fields are the sums of Lennard-Jones (LJ) and electrostatic contributions. Water molecules are described by the well documented SPC/E model ${ }^{19}$ with a LJ core centered on the oxygen $\left(\sigma=3.1655 \AA, \epsilon=0.1554 \mathrm{kcal} \mathrm{mol}^{-1}\right)$ and partial charges on the oxygen and hydrogen atoms $\left(r_{\mathrm{OH}}=1 \AA, \theta_{\mathrm{HOH}}=109.47^{\circ}, q_{\mathrm{H}}=+0.4238\right)$.

On the other hand, information on LJ parameters for the ions, especially $\mathrm{Eu}^{3+}$, is quite sparse in the literature. To describe $\mathrm{EuCl}_{3}$ solutions, we use the parameters by Ribeiro 9 : $\sigma_{\mathrm{Eu}^{3+}}=1.78 \AA$, $\sigma_{\mathrm{Cl}^{-}}=4.42 \AA, \epsilon_{\mathrm{Eu}^{3+}}=5 \mathrm{kcal} \mathrm{mol}^{-1}, \epsilon_{\mathrm{Cl}^{-}}=0.118 \mathrm{kcal} \mathrm{mol}^{-1}$. Cross-interactions are computing using Lorentz-Berthelot mixing rules.

\subsubsection{Simulations}

We use OpenMM 7.2.0 $0^{20}$ to simulate the dynamics of $N_{i}=180 / 540 / 5544$ particles $\left(\mathrm{Eu}^{3+} / \mathrm{Cl}^{-} / \mathrm{H}_{2} \mathrm{O}\right)$ at 298.15 Kelvin and $10^{5}$ Pascal in NPT ensemble. We use Langevin dynamics with a time step of 2 fs to simulate the evolution at constant temperature. To simulate constant pressure, we use a Monte Carlo Metropolis algorithm: Every 50 ps, a change in the volume of the periodic cell is accepted or refused based on the temperature and cost in internal energy. The LJ potentials have been truncated at $10 \AA$ and shifted. The tails have been added using the textbook meanfield procedure ${ }^{21}$. The electrostatic interactions within the periodic boundary (tin-foil; $\epsilon=\infty$ ) conditions have been calculated using the Particle Mesh Ewald techniques ${ }^{22}$ with a short range cut-off at $10 \AA$ and an error tolerance on the forces of 0.00005 . After 10 ns equilibration, a $140 \mathrm{~ns}$ long trajectory has been analyzed every $1 \mathrm{ps}$. For that $1.8 \mathrm{~mol} / \mathrm{kg}$ molal density, the obtained average molar densities are $\rho_{i}=1.68 / 5.04 / 51.70 \mathrm{~mol} / \mathrm{l}$. The fluctuations of the cell volume yield the value $\chi=0.0395$ for the normalized compressibility $\chi=\left.\left(\frac{\partial \rho}{\partial \beta P}\right)\right|_{\rho_{i} / \rho}$ where $\rho$ is the total density and $\beta=1 / k T$. The fluctuations of the total water dipolar moment of the simulation cell yield 24.1 for the dielectric constant.

The 6 PDFs are constructed from each of the 140000 configurations using distance histograms 
up to $L / 2$. For the 3 ion-ion functions, the situation is simple since the PDFs $g_{i j}(r)$ between spherical ions depend on the separation $r$ only. On the other hand, the complete description of the anisotropic ion-water and water-water correlations requires a special treatment. Rather than manipulating costly angle histograms (which would require 2 and 5 Euler angles for ion-water and water-water correlations, respectively), we project the complete $g_{i j}(r, \Omega)$ on a basis of rotational invariants and accumulate the projections $g_{i j}^{m n}{ }_{\mu \nu}(r)$ that depend on the separation $r$ only. This procedure, which has been proven recently to be very efficient for a complete description of angular correlations in pure water $\frac{12}{12}$, is extended here to a 3 component electrolyte system. The origin of the $\mathrm{H}_{2} \mathrm{O}$ molecule is chosen on the oxygen atom and its main axis points along the dipole. The

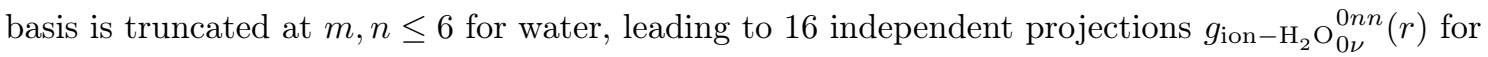
both ion-water PDFs and 1226 independent $g_{\mathrm{H}_{2} \mathrm{O}-\mathrm{H}_{2} \mathrm{O}}^{m n l}{ }_{\mu \nu}(r)$.

\section{Results and discussion}

\subsection{Simulation of pair distribution functions and partial structure fac- tors - mixed MD/HNC integral equation}

Figure 1 1 illustrates a typical snapshot of the MD trajectory. It is observed that both $\mathrm{Eu}^{3+}$ and $\mathrm{Cl}^{-}$ions are homogeneously distributed in the water matrix. As expected, oxygen atoms are oriented toward the $\mathrm{Eu}^{3+}$ ions whereas hydrogen atoms are oriented toward the $\mathrm{Cl}^{-}$ions. From the long trajectory, center of mass-center of mass projections $g_{i j 00}^{000}(r)$ (the $g_{i j}(r, \Omega)$ averaged over the orientation $\Omega$ ), noted more simply $g_{i j}(r)$, are calculated and presented, for 6 PDFs, in Fig. 11b. Owing to the limited size of the periodic box $(L=56 \AA)$, in average the theoretically simulated PDFs (as demonstrated in Fig. 1b) are partial, to the $r$ region below the cut-off $L / 2$, and are missing non-negligible tails. As a result, the Fourier transformed signals i.e., partial structure factors $S_{i j}(q)$ will have undesired oscillations and uncontrolled zero- $q$ values in the low $q$ region. Therefore, to get the appropriate simulation curve, one needs extending the simulation data with an ad-hoc procedure which combines MD and HNC. Further, we would like to remind the reader that the MD data at short distances $r<L / 2$ are not exact and are subject to systematic finite-size corrections. In particular, it is well known since Percus-Lebowitz ${ }^{23}$ that, within the NVT or NPT ensemble, the PDF $g_{i j}(r)$ does not reach the correct asymptotic value 1 at large separation but rather 1- $\left\lceil S_{i j}(q) / \sqrt{\left(N_{i} N_{j}\right)}\right\rceil$. The shift in $1 / N$ may appear very small but, when integrated on a large part of the simulation cell volume, it induces a non-negligible artefact to $g_{i j}(r)$. It is thus necessary to first clean the simulation data from these shifts before use. This problem is central in the determination of the so-called Kirkwood-Buff integrals, a rich literature ${ }^{24|25| 26}$ exists, a future paper will propose special procedures for $N V T$ or $N P T$ simulations of mixtures. We would 
just mention here that the situation is a vicious circle because, in order to calculate the correct integral $S_{i j}$, one must first clean the MD $g_{i j}$ by requiring the knowledge of $S_{i j}$. In a first step, we will ignore these corrections and apply the MD/HNC mixed equation with brute MD data. The obtained values for the $S_{i j}$ will then be used to clean the MD PDFs and the procedure will be repeated until self-consistency is reached in a couple of iterations.

The formally exact integral equation can be written like the following:

$$
g_{i j}(r, \Omega)=\exp \left[-\beta \nu_{i j}(r, \Omega)+h_{i j}(r, \Omega)-c_{i j}(r, \Omega)+b_{i j}(r, \Omega)\right]
$$

where $h_{i j}=g_{i j}-1$ and $c_{i j}$ are the total and direct correlation functions, respectively, linked through the molecular $\mathrm{OZ}$ equation. The $b_{i j}$ are the unknown bridge functions, neglected by the HNC approximation.

The mixed MD/HNC integral equation becomes in projection notation:

$$
\begin{gathered}
g_{i j \mu \nu}^{m n l}(r)=g_{i j}^{M D^{m n l}}(r) ; r<r_{\max } \\
b_{i j \mu \nu}^{m n l}(r)=0 ; r>r_{\max }
\end{gathered}
$$

The cut-off distance $r_{\max }$ is obviously chosen below the available $L / 2$ value. It is a priori expected and a posteriori verified that the bridge functions, which are shorter range than the PDFs, are indeed negligible beyond $r_{\max }$.

The numerical resolution of the multi-component molecular integral equation follows a standard, well documented iterative procedure, using algorithms which improves step by step the projections of the functions $\gamma_{i j}=h_{i j}-c_{i j}$ in the whole $r$-space. Briefly, an elementary cycle goes back and forth between $r$ and $q$-spaces by performing numerical Hankel-Fourier (HF) transforms of the $c_{i j}(r)$ projections and inverse $\mathrm{HF}$ of the $\hat{\gamma} i j(q)$ ones. The closure is applied in the $r$-space while the $\mathrm{OZ}$ equation is better written as simple algebraic products between projections in the $q$-space. The choice of a starting reasonable guess function, so important to avoid instantaneous divergence of the iteration, requires a special comment for the present $\mathrm{EuCl}_{3}$ systems. When a full $\mathrm{HNC}$ solution is the objective, it is standard routine to increase gradually the interaction coupling (say, the inverse temperature $\beta$ or the valencies and partial charges) and start the resolution of a given coupling from the final solution of the previous, weaker one. When the full HNC solution exists and the $\mathrm{MD} / \mathrm{HNC}$ solution is the objective (as in pure water or monovalent $\mathrm{NaCl}$ electrolytes), it is possible in the same manner to inject gradually the MD data (say, by increasing $r_{\max }$ from 0 ). But here, as already mentioned (in the introduction), the studied $\mathrm{Eu}^{3+} / \mathrm{Cl}^{-} / \mathrm{H}_{2} \mathrm{O}$ system has no 
HNC solution (the system lies in the HNC forbidden region of the phase diagram). So, the search of a reasonable initialization solution which guarantees subsequent convergence is far from trivial.

Our successful recipe follows different successive steps: Each of the MD $h_{i j}$ projections is fitted with a damped oscillating function $A=\frac{e^{-\alpha r}}{r} \sin q(r-d)$ on the domain $[17 \AA, 27 \AA]$ and so artificially extended up to $r_{\max }=50 \AA$. Each $h_{i j}{ }_{\mu \nu}^{m n l}(r)$ is numerically HF transformed using the upper boundary $r=r_{\max }$. As expected, the resulting $\hat{h}_{i j}{ }_{\mu \nu}^{m n l}(q)$ display inconsistent behaviour at low $q$ due to the lack of correct tails in the PDFs, the previous long range extension playing only the role of narrowing this unreachable $q$ region. The situation is even worse for the corresponding $\hat{\gamma}_{i j \mu \nu}^{m n l}(q)$ projections derived from the OZ equation which schematically reads as $\hat{\gamma}=\frac{\hat{h}^{2}}{1+\hat{h}}$ and transforms inconsistent $\hat{h}_{i j}$ into $\hat{\gamma}_{i j}$ full of divergences in the low $q$-region. Nevertheless, the different $\hat{\gamma}_{i j}{ }_{\mu \nu}^{m n l}(q)$ projections keep a reasonable shape at larger $q$. The next step consists to extrapolate each of them on the domain $\left[0,0.6 \AA^{-1}\right]$ using a 4 th order polynomial fitted on the domain $\left[0.6 \AA^{-1}, 1.4 \AA^{-1}\right]$. The known divergences in $1 / q^{2}$ and $1 / q$ due to the ion-ion and ion-dipole interactions, respectively, are handled in the usual way. Lastly, an inverse HF transform leads to a desired guess for the $\gamma_{i j}{ }_{\mu \nu}^{m n l}(r)$. When the standard resolution of the mixed integral equation (eq,1) (still for the moment with $r_{\max }=50 \AA$ ) is initiated from this guess, surprisingly, no divergence is observed at low $q$ in the first cycle and convergence can be reached in a few iterations. Once the solution has been obtained for the artificially extended MD data, it is then a routine to decrease gradually the cut off $r_{\max }$ from $50 \AA$ down to a value below $L / 2$, such that only the bare MD data are imposed. The final MD/HNC solution is of course independent of the detailed recipe followed to reach it.

The MD/HNC PDFs $g_{i j}$ obtained with $r_{\max }=25 \AA$ are displayed in Fig. 2 and compared to the bare MD data. As expected, the MD/HNC curves do not exhibit any discontinuity at $r_{\max }$, reproduce in great detail the bare $\mathrm{MD}$ ones in the domain $\left[r_{\max }, L / 2\right]$ and extend them nicely beyond $L / 2$.

From Fig. 1 $1 \mathrm{~b}$, the first maximum of PDFs between ions-ions, ions- $\mathrm{H}_{2} \mathrm{O}$ and $\mathrm{H}_{2} \mathrm{O}-\mathrm{H}_{2} \mathrm{O}$ in 1.8 $\mathrm{mol} / \mathrm{kg}$ aqueous solution are found about: $r_{\mathrm{Eu}^{+3} \mathrm{Eu}^{+3}}=8.5 \AA, r_{\mathrm{Cl}^{-} \mathrm{Cl}^{-}}=4.7 \AA, r_{\mathrm{Eu}^{+3} \mathrm{Cl}^{-}}=5.3 \AA$, $r_{\mathrm{Eu}^{+3} \mathrm{H}_{2} \mathrm{O}}=2.3 \AA, r_{\mathrm{Cl}^{-} \mathrm{H}_{2} \mathrm{O}}=3.2 \AA$, and $r_{\mathrm{H}_{2} \mathrm{OH}_{2} \mathrm{O}}=2.7 \AA$. By comparing these values with the nearest neighbouring distance in $\mathrm{EuCl}_{3}$ salt and size of water molecule, we exclude the possibility that identical ions form pairs. Further, any pair of identical ions are found to separated at-least by two water molecules and by an oppositely charged ion.

Like for $r$-space, the same type of expansion exists in the $q$-space. We will focus here on the center of mass-center of mass partial structure factors $S_{i j}(q)$ defined from the 3D Fourier transform of the $g_{i j}(r)$ : 

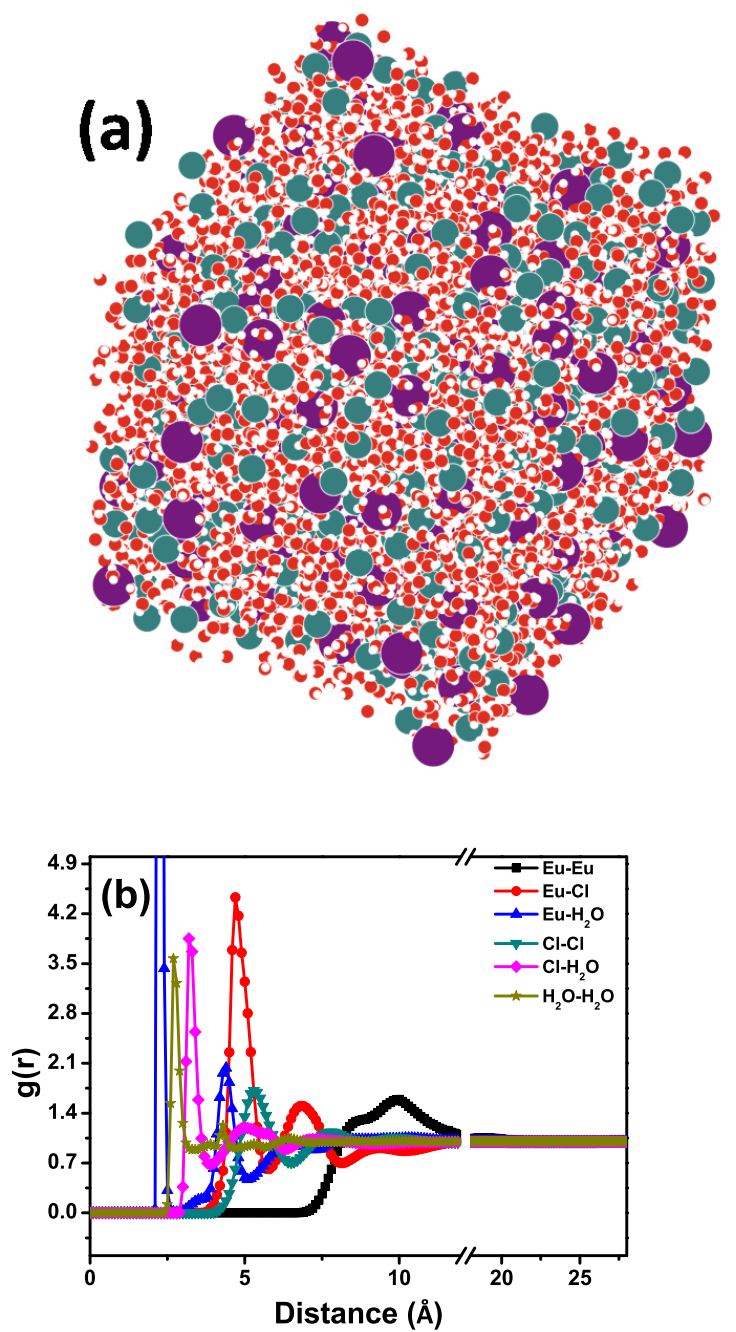

Figure 1: (a) A typical snapshot of the MD trajectory. Color code: Turquoise: $\mathrm{Eu}^{+3}$, purple: $\mathrm{Cl}^{-1}$, red: oxygen, white: hydrogen. (b) Bare MD center of mass - center of mass pair distribution functions $g_{i j}(r)$ in $\mathrm{EuCl}_{3} 1.8 \mathrm{~mol} / \mathrm{kg}$ aqueous solution.

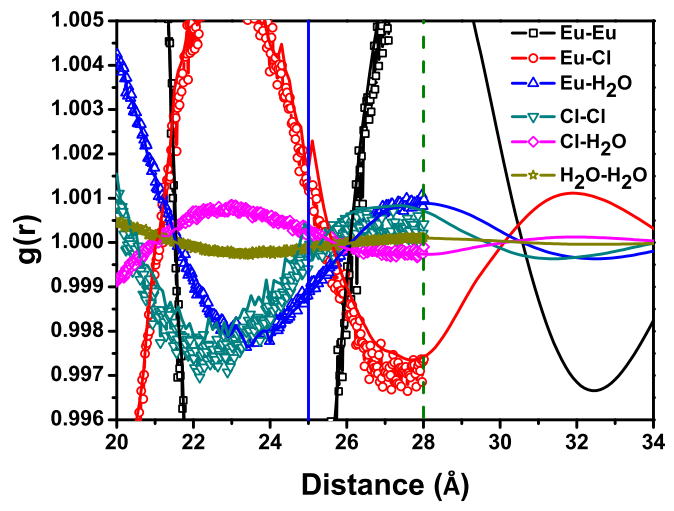

Figure 2: Comparison of the bare MD (symbols+lines) and MD/HNC (lines) PDFs $g_{i j}(r)$ in $\mathrm{EuCl}_{3}$ $1.8 \mathrm{~mol} / \mathrm{kg}$ aqueous solution around the cutoff distance $r_{\max }=25 \AA . r_{\max }$ and $L / 2$ are indicated by solid and dashed lines, respectively. 

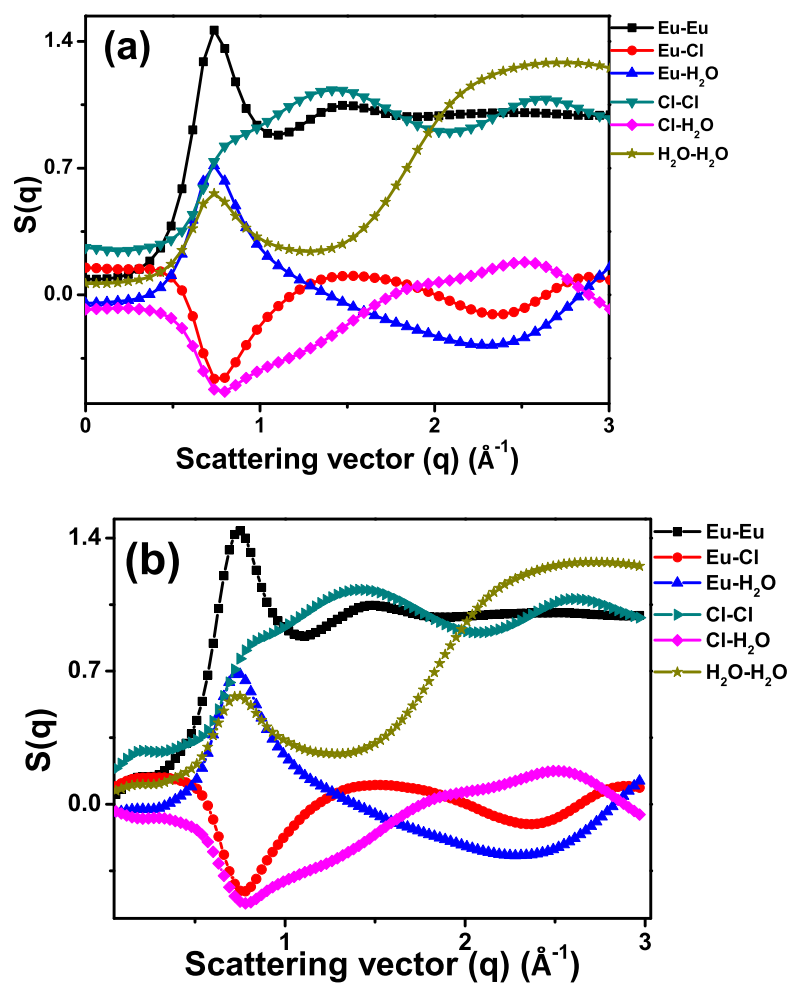

Figure 3: (a) $\mathrm{MD} / \mathrm{HNC}$ partial structure factors $S_{i j}(q)$ of $\mathrm{EuCl}_{3} 1.8 \mathrm{~mol} / \mathrm{kg}$ aqueous solution. (b) Pure MD partial structure factors $S_{i j}(q)$ of $\mathrm{EuCl}_{3} 1.8 \mathrm{~mol} / \mathrm{kg}$ aqueous solution. 


$$
S_{i j}(q)=\delta_{i j}+\sqrt{\rho_{i} \rho_{j}} \int_{0}^{+\infty}\left[g_{i j}(r)-1\right] \frac{\sin (q r)}{q r} 4 \pi r^{2} d r
$$

The obtained partial structure factors $S_{i j}(q)$ are displayed in Fig. 3 a. With the mixed $\mathrm{MD} / \mathrm{HNC}$ integral equation, full $q$ range is described precisely. The MD/HNC values at $q=0$ are $S_{\mathrm{Cl}^{-} \mathrm{Cl}^{-}}=0.26, S_{\mathrm{Cl}^{-} \mathrm{H}_{2} \mathrm{O}}=-0.079$ and $S_{\mathrm{H}_{2} \mathrm{O} \mathrm{H}} \mathrm{O}=0.062$. Note that the electroneutrality conditions $S_{\mathrm{Cl}^{-} \mathrm{Cl}^{-}}=\sqrt{3} S_{\mathrm{Eu}^{+3} \mathrm{Cl}^{-}}=3 S_{\mathrm{Eu}^{+3} \mathrm{Eu}^{+3}}$ and $S_{\mathrm{Cl}^{-} \mathrm{H}_{2} \mathrm{O}}=\sqrt{3} S_{\mathrm{Eu}^{+3} \mathrm{H}_{2} \mathrm{O}}$ are rigorously satisfied. The corresponding osmotic compressibility $\chi_{o s m}=\left.\left(\frac{\partial \rho_{\text {ion }}}{\partial(\beta P)}\right)\right|_{\mu\left(\mathrm{H}_{2} \mathrm{O}\right)}(\mu$ represents the chemical potential) is equal to $4 / 3 S_{\mathrm{Cl}^{-} \mathrm{Cl}^{-}}=0.35$. The total scattered intensity is then trivially derived from these 6 cross contributions (see subsection 4.4. In contrast, structure factors of pure MD simulated data (Fig. 3b) produce spurious oscillations at low- $q$ along with a wrong estimation of osmotic compressibility.

\subsection{Water-water structure factor with and without the presence of $\mathrm{EuCl}_{3}$}

Having calculated the right partial structure factors (in the entire $q$-range), we shall examine the water-water structure factors with and without the presence of $\mathrm{EuCl}_{3}$ salt. They are plotted and compared in Fig. 4 along with the experimental partial structure factor of pure water recorded both at lab and ID02 beamline (ESRF). As reported earlier ${ }^{27}$, both experimental and theoretical signals show characteristic broad bands of pure water centered at about 2 and $3 \AA^{-1}$, whereas the addition of $\mathrm{EuCl}_{3}$ generates an additional correlation peak in the partial structure factor of water at about $0.75 \AA^{-1}$ along with the merging of previously observed two broad bands of water. Further, the position of correlation peak is found to occur around the same position as observed as for the partial structure factors of $\mathrm{Eu}^{3+}-\mathrm{Eu}^{3+}, \mathrm{Cl}^{-}-\mathrm{Cl}^{-}$and $\mathrm{Eu}^{3+}-\mathrm{H}_{2} \mathrm{O}$ (see, Fig. 3 3). The modified water signal indicates that, first, the structure of water gets modified in presence of $\mathrm{EuCl}_{3}$ salt. Second, as reported ${ }^{13}$, there would be an emergence of long range correlation between the water molecules participating in the first shell around the cation/anion. As a result, the new correlation peak is appearing, at low $q$, in the partial structure factor of water in presence of $\mathrm{EuCl}_{3}$ salt.

\subsection{Experimental X-ray scattering of $\mathrm{EuCl}_{3}$ aqueous solutions}

Further, Fig. 5 shows the experimental X-ray scattering signal of $\mathrm{EuCl}_{3}$ aqueous solutions with varying concentrations i.e. from 0.15 to $1.8 \mathrm{~mol} / \mathrm{kg}$. Irrespective of the concentration of $\mathrm{EuCl}_{3}$ aqueous solutions, the experimental patterns demonstrate a correlation peak and an oscillating signal beyond $1.5 \AA^{-1}$. Upon dilution, the center of the correlation peak shifts from $q \simeq 0.7$ to $0.3 \AA^{-1}$ and the oscillating signal beyond $1.5 \AA^{-1}$ is progressively dominated by the broad bands characteristic to the water signal (see Fig. 4). As confirmed below, the observed correlation peak 


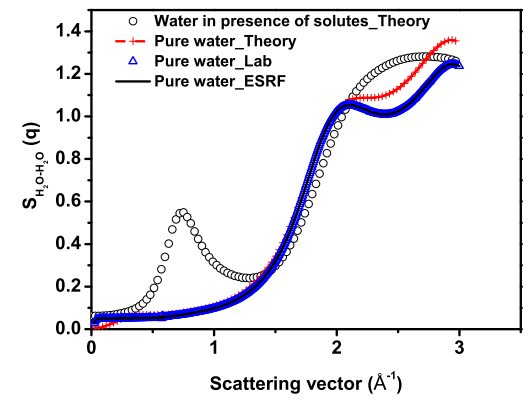

Figure 4: Theoretical water-water structure factors with and without the presence of $\mathrm{EuCl}_{3}$ are compared with experimental structure factor of pure water recorded both at lab and ID02 beamline (ESRF).

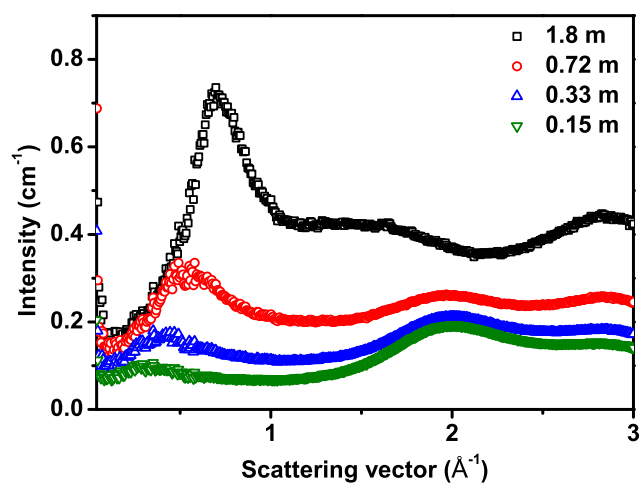

Figure 5: Experimental X-ray scattering signal of $\mathrm{EuCl}_{3}$ aqueous solutions.

at low $q$, for all solutions, is attributed to the predominant long ranged interactions between the cation-cation i.e. $\mathrm{Eu}^{3+}-\mathrm{Eu}^{3+}$.

To follow the real space correlation between the $\mathrm{Eu}^{3+}-\mathrm{Eu}^{3+}[13$, the position of the center of correlation-peak is plotted, in Fig. 6, as a function of the concentration of $\mathrm{EuCl}_{3}$ aqueous solutions to the power one third $\left(\mathrm{c}^{1 / 3}\right)$. From the Fig. 6, as expected, upon dilution of the $\mathrm{EuCl}_{3}$ aqueous solution the distance between $\mathrm{Eu}^{3+}-\mathrm{Eu}^{3+}$ increases and reaches the maximum value for the lowest concentration. The $\mathrm{c}^{1 / 3}$ behavior illustrates the strong effective repulsion between the $\mathrm{Eu}^{3+}$ ions which tend to maximize the distance between neighbors.

\subsection{Comparison between the experimental and theoretical X-ray scat- tering signals of $1.8 \mathrm{~mol} / \mathrm{kg} \mathrm{EuCl}_{3}$ aqueous solution}

The various possible structural correlation components (simulated for the highest concentration of $\mathrm{EuCl}_{3}$ aqueous solution i.e., $1.8 \mathrm{~mol} / \mathrm{kg}$ ) should be introduced into the following equation to determine the total X-ray scattering signal. 


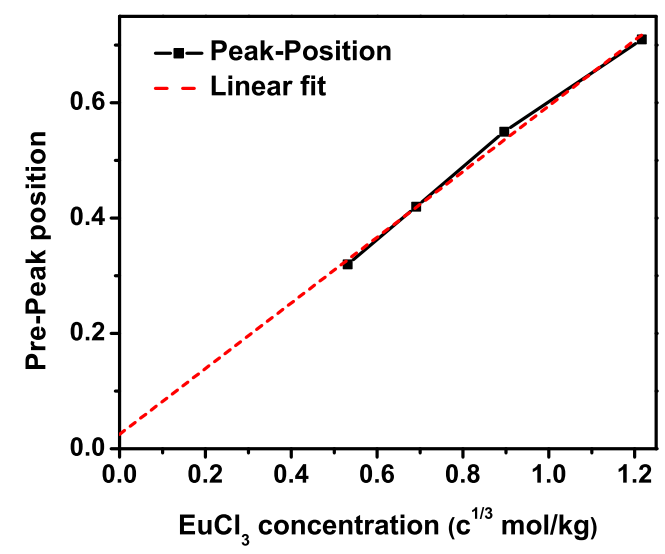

Figure 6: Pre-peak position as a function of concentration $\left(\mathrm{c}^{1 / 3}\right)$.

$$
I(q)=\Sigma_{i} \Sigma_{j} \sqrt{\rho_{i} \rho_{j}} f_{i}(q) f_{j}(q) S_{i j}(q)
$$

where $f_{i}(q)$ stands for the amplitude factor of particle i. Note that eq. 5 which involves the center of mass-center of mass projections only, implicitly assumes that each particle scatters the X-rays as spheres. For ions, values of $f_{i}(q)$, as a function of scattering vectors, were derived by considering the electronic density of $\mathrm{Eu}^{3+}$ and $\mathrm{Cl}^{-}$homogeneously distributed in a sphere with the radius $0.48 \AA$ and $0.63 \AA$, respectively, whereas for water molecules values were retrieved from Morin 28 .

As a result, partial and total theoretical X-ray scattering signals of $1.8 \mathrm{~mol} / \mathrm{kg} \mathrm{EuCl}$ aqueous solution were obtained. Subsequently, experimental, partial and total theoretical X-ray scattering signals of $1.8 \mathrm{~mol} / \mathrm{kg} \mathrm{EuCl} 3$ aqueous solution are plotted in Fig. 7. The shape of the total theoretical X-ray scattering signal is closely matching with the recorded signal. But, there is a slight discrepancy in the intensity observed between the experimental and theoretical signals along with a shift in the position of theoretical curve above $1.5 \AA^{-1}$. As stated above, the detail of the contributions from each partial intensity factor confirms the correlation peak is dominated by the $\mathrm{Eu}^{3+}-\mathrm{Eu}^{3+}$ interactions whereas at high $q$, both $\mathrm{Eu}^{3+}-\mathrm{Eu}^{3+}$ and $\mathrm{H}_{2} \mathrm{O}-\mathrm{H}_{2} \mathrm{O}$ interactions contribute to the signals. These result confirms that the mere LJ potentials and electrostatic interactions are sufficient to reproduce the under-saturated experimental signal. Contrarily to other systems, there is no need of incorporation of ion pairs, pre-nucleation clusters or close-packed hydrated cation models ${ }^{13 / 29|30| 31}$ where simple LJ and electrostatic interactions are explicitly completed by specific ion-ion or ion-water correlations. 


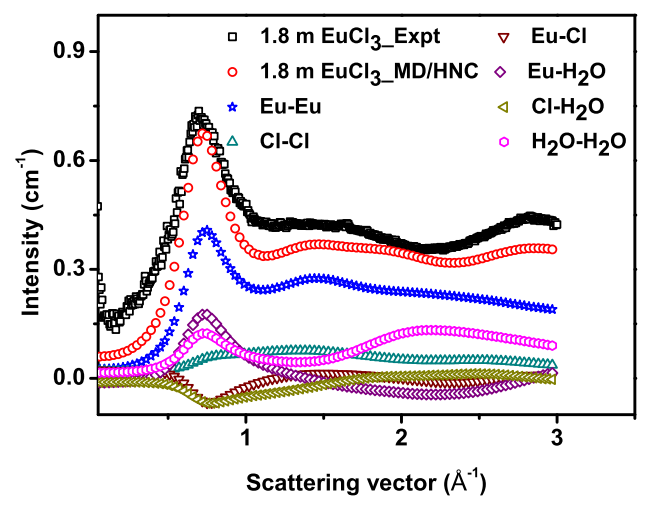

Figure 7: Experimental, partial and total theoretical X-ray scattering signals of $1.8 \mathrm{~mol} / \mathrm{kg} \mathrm{EuCl} \mathrm{E}_{3}$ aqueous solution.

\section{Summary}

$\mathrm{X}$-ray scattering signal of $\mathrm{EuCl}_{3}$ aqueous solutions were recorded as a function of varying concentrations. Upon dilution, the correlation-peak at low- $q$ is dominated by the long-ranged $\mathrm{Eu}^{3+}-\mathrm{Eu}^{3+}$ interactions mediated by water molecules. The under-saturated $1.8 \mathrm{~mol} / \mathrm{kg} \mathrm{EuCl} \mathrm{El}_{3}$ aqueous solution was compared with the coupled $\mathrm{MD} / \mathrm{HNC}$ model. A good consistency is found between the experimental and theoretical patterns, merely using the simple LJ and electrostatic interaction parameters. The comparison of both signals demonstrate that contrarily to other systems $\left(\mathrm{CaCO}_{3}\right.$, $\mathrm{MgCl}_{2}$, etc.), the incorporation of ion pairs or pre-nucleation clusters is not needed.

\section{Acknowledgements}

RKR acknowledges support by the French National Research Agency (ANR) as part of the "Investissements d'Avenir" program (LabexNanoSaclay, reference: ANR-10-LABX-0035) and by FP7 Marie Curie funded Enhanced Eurotalents programme at the CEA. DC acknowledge a public grant overseen by ANR, project DIAMONS ANR-14-CE08-0003. Further, we would like to acknowledge the beamtime at ID02 beamline (ESRF).

\section{References}

[1] Yoreo, J. J. D.; Gilbert, P. U. P. A.; Sommerdijk, N. A. J. M.; Penn, R. L.; Whitelam, S.; Joester, D.; Zhang, H.; Rimer, J. D.; Navrotsky, A.; Banfield, J. F.; Wallace, A. F.; 
Michel, F. M.; Meldrum, F. C.; Cölfen, H.; Dove, P. M. Crystallization by particle attachment in synthetic, biogenic, and geologic environments. Science 2015, 349, aaa6760.

[2] Xie, F.; Zhang, T. A.; Dreisinger, D.; Doyle, F. A critical review on solvent extraction of rare earths from aqueous solutions. Miner. Eng. 2014, 56, 10-28.

[3] Kononov, L. O. Chemical reactivity and solution structure: on the way to paradigm shift? $R S C A d v .2015,5,46718-46734$.

[4] Ballard, A. J.; Dellago, C. Toward the mechanism of ionic dissociation in water. J. Phys. Chem. B 2012, 116, 13490-13497.

[5] Marques, M. A.; de Barros Marques, M.; Cabaço, M. I.; Gaspar, A.; de Almeida, M. Intermediate range order in concentrated aqueous solutions of copper nitrate. X-ray diffraction and Raman investigations. J. Mol. Liq. 2004, 110, 23-31.

[6] Coste, A.; Poulesquen, A.; Diat, O.; Dufrêche, J.-F.; Duvail, M. Investigation of the structure of concentrated $\mathrm{NaOH}$ aqueous solutions by combining molecular dynamics and wide-angle X-ray scattering. J. Phys. Chem. B 2019, 123(24), 5121-5130.

[7] Prins, J. A. Molecular arrangement and X-ray diffraction in ionic solutions. J. Chem. Phys. 1935, 3, 72-80.

[8] Degréve, L.; da Silva, F. L. B. Structure of concentrated aqueous NaCl solution: A Monte Carlo study. J. Phys. Chem. 1999, 110, 3070.

[9] Ribeiro, M. C. C. Intermediate-range order and collective dynamics in an aqueous solution of trivalent cations. Phys. Rev. B 2006, 73, 014201(1-7).

[10] Belloni, L. Finite-size corrections in numerical simulation of liquid water. J. Phys. Chem. 2018, $149,094111$.

[11] Verlet, L. Computer "experiments" on classical fluids. II. Equilibrium correlation functions. Phys. Rev. 1968, 163, 201.

[12] Belloni, L. Exact molecular direct, cavity, and bridge functions in water system. J. Chem. Phys. 2017, 147, 164121.

[13] Ribeiro, M. C. C. Intermediate-range order in aqueous solutions of salts: a systematic computer simulation study. J. Phys.: Condens. Matter 2005, 17, 453-467.

[14] Fleury, B.; Neouze, M.-A.; Guigner, J.-M.; Menguy, N.; Spalla, O.; Gacoin, T.; Carriere, D. Amorphous to crystal conversion as a mechanism governing the structure of luminescent $\mathrm{YVO}_{4}$ : Eu nanoparticles. ACS Nano 2014, 8(3), 2602-2608. 
[15] Binnemans, K.; Jones, P. T.; Blanpain, B.; Gerven, T. V.; Yang, Y.; Walton, A.; Buchert, M. Recycling of rare earths: a critical review. J. Clean. Prod. 2013, 51, 1-22.

[16] Taché, O.; Rouzière, S.; Joly, P.; Amara, M.; Fleury, B.; Thill, A.; Launois, P.; Spalla, O.; Abécassis, B. MOMAC: a SAXS/WAXS laboratory instrument dedicated to nanomaterials. J. Appl. Crystallogr. 2016, 49, 1624-1631.

[17] Zemb, T.; Taché, O.; Né, F.; Spalla, O. Improving sensitivity of a small angle X-ray scattering camera with pinhole collimation using separated optical elements. Rev. Sci. Instrum. 2003, $74(4), 2456-2462$.

[18] Narayanan, T.; Sztucki, M.; Van Vaerenbergh, P.; Léonardon, J.; Gorini, J.; Claustre, L.; Sever, F.; Morse, J.; Boesecke, P. A multipurpose instrument for time-resolved ultra-smallangle and coherent X-ray scattering. J. Appl. Crystallogr. 2018, 51(6), 1511-1524.

[19] Berendsen, H. J. C.; Grigera, J. R.; Straatsma, T. P. The missing term in effective pair potentials. J. Phys. Chem. 1987, 91, 6269-6271.

[20] Eastman, P.; Swails, J.; Chodera, J. D.; McGibbon, R. T.; Zhao, Y.; Beauchamp, K. A.; Wang, L.-P.; Simmonett, A. C.; Harrigan, M. P.; Stern, C. D.; Wiewiora, R. P.; Brooks, B. R.; Pande, V. S. OpenMM 7: Rapid development of high performance algorithms for molecular dynamics. PLoS Comput. Biol. 2017, 13(7), e1005659.

[21] Allen, M. P.; Tildesley, D. J. Computer simulation of liquids, reprinted with corrections ed.; Oxford University Press Inc., Newyork, 1989.

[22] Essmann, U.; Perera, L.; Berkowitz, M. L.; Darden, T.; Lee, H.; Pedersen, L. G. A smooth particle mesh Ewald method. J. Chem. Phys. 1995, 103(19), 8577-8593.

[23] Lebowitz, J. L.; Percus, J. K. Long-Range correlations in a closed system with applications to nonuniform fluids. Phys. Rev. 1961, 122, 1675.

[24] Perera, A.; Zoranic, L.; Sokolic, S.; Mazighi, R. A comparative molecular dynamics study of water-methanol and acetone-methanol mixtures. J. Mol. Liq. 2011, 159, 52-59.

[25] Krüger, P.; Schnell, S. K.; Bedeaux, D.; Kjelstrup, S.; Vlugt, T. J. H.; Simon, J. Kirkwood-Buff integrals for finite volumes. J. Phys. Chem. Lett. 2013, 4, 235-238.

[26] Cortes-Huerto, R.; Kremer, K.; Potestio, R. Communication: Kirkwood-Buff integrals in the thermodynamic limit from small-sized molecular dynamics simulations. J. Chem. Phys. 2016, $145,141103$.

[27] Soper, A. K. Supercooled water: Continuous trends. Nat. Mater. 2014, 13, 671-673. 
[28] Morin, L. R. M. Molecular form factors and photon coherent scattering cross sections of water. J. Phys. Chem. Ref. Data 1982, 11(4), 1091-1098.

[29] Smeets, P. J. M.; Finney, A. R.; Habraken, W. J. E. M.; Nudelman, F.; Friedrich, H.; Laven, J.; Yoreo, J. J. D.; Rodger, P. M.; Sommerdijk, N. A. J. M. A classical view on nonclassical nucleation. Proc. Natl. Acad. Sci. U.S.A 2017, 114 (38), E7882-E7890.

[30] Gebauer, D.; Völkel, A.; Cölfen, H. Stable prenucleation calcium carbonate clusters. Science 2008, 322, 1819 .

[31] Gaspar, A. M.; Marques, M. A.; Cabaço, M.; de Barros Marques, M. I.; Buslaps, T.; Honkimaki, V. X-Ray diffraction investigations of concentrated aqueous solutions of calcium halides. J. Mol. Liq. 2004, 110, 15-22. 